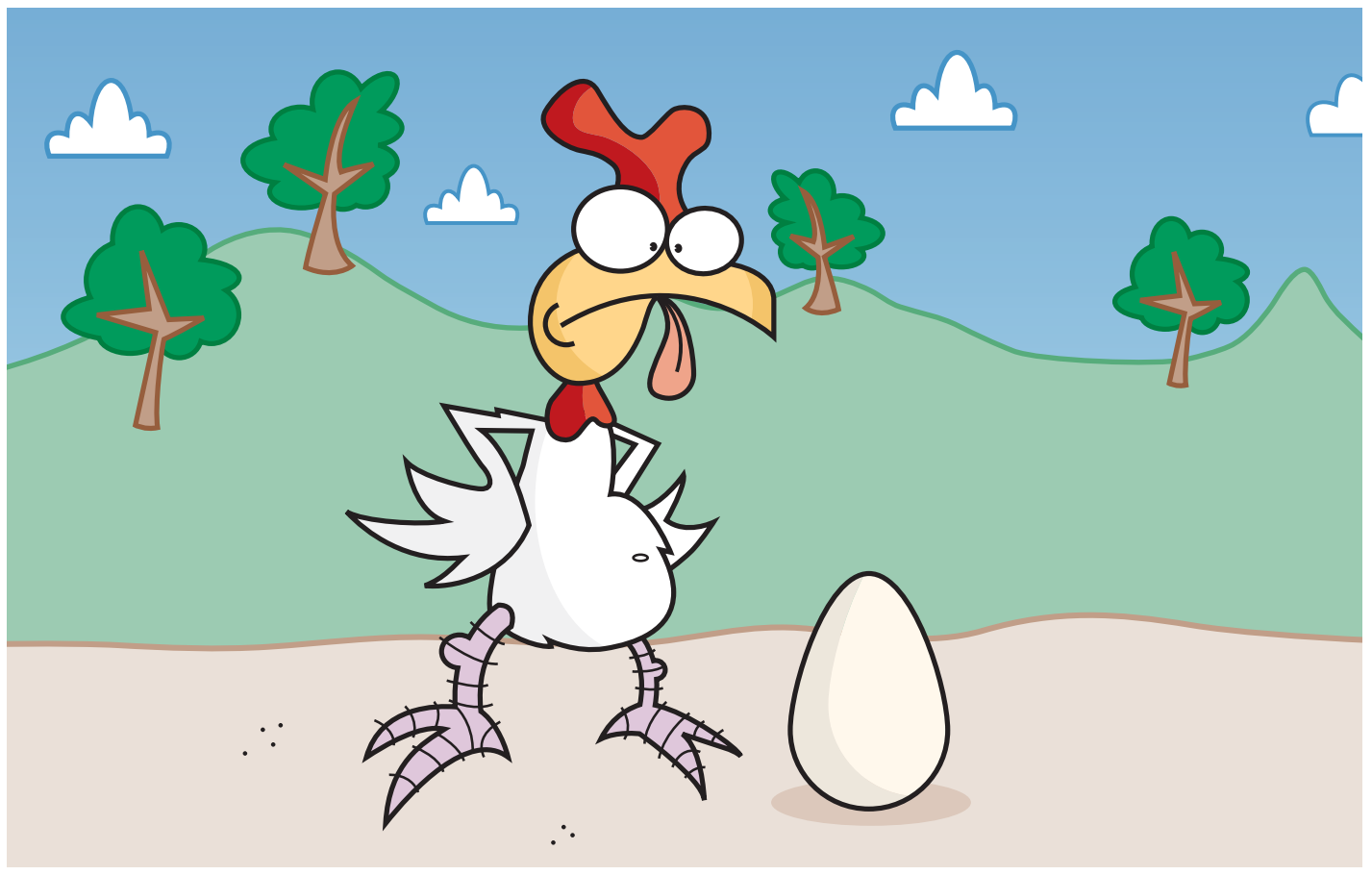

B CELLS

\title{
What comes first: DNA cleavage or UNG?
}

A new study by Honjo and colleagues, recently published in Science, adds to the controversy about the role of activation-induced cytidine deaminase (AID) in class-switch recombination (CSR) - the process by which antibodies switch their isotype from IgM to IgG, IgA or IgE by recombination at the heavy-chain (IgH) loci. AID is known to be required for the doublestranded DNA breaks that allow recombination, but the way in which it achieves this is hotly debated.

The most widely accepted model (see Further reading) proposes that AID deaminates cytosine residues to uracil residues in immunoglobulin-gene DNA; the uracil residues are then removed by the DNA base-excision repair enzyme uracil-DNA glycosylase (UNG), resulting in strand breaks. However, Honjo and colleagues have proposed an alternative model on the basis of similarities between AID and the mRNA-editing enzyme APOBEC1 - in which AID deaminates an unknown mRNA such that it encodes a novel endonuclease that cleaves the DNA. By looking at the temporal relationship between DNA breaks and UNG activity, they now put forward further evidence in favour of the RNA-editing model.

In these experiments, phosphorylated histone $\mathrm{H} 2 \mathrm{AX}(\gamma-\mathrm{H} 2 \mathrm{AX})$, which forms next to doublestranded DNA breaks, was used as a marker for cleavage sites involved in CSR. When a mouse lymphoma cell line was stimulated to switch to IgA production, $\gamma-\mathrm{H} 2 \mathrm{AX}$ accumulated at the IgH loci. Expression of the UNG inhibitor Ugi by these cells reduced the level of CSR to IgA but did not affect
$\gamma-\mathrm{H} 2 \mathrm{AX}$ accumulation. Because $\gamma-\mathrm{H} 2 \mathrm{AX}$ focus formation at the IgH loci does not depend on UNG, but CSR is reduced in the absence of UNG, this indicates that UNG has a role in CSR downstream of DNA cleavage, which contradicts the DNAdeamination model. In support of this, there was no difference in the IgH mutation rate between cells expressing or not expressing Ugi; because mutation results from DNA cleavage during CSR, it seems probable that UNG is not required for DNA cleavage.

UNG mutants that lack UNG catalytic activity but retain DNA-binding activity were shown to recover wild-type levels of CSR in UNG-deficient B cells. The importance of UNG for CSR in the absence of its catalytic activity indicates that it might have a structural role. Honjo and colleagues elaborate on their RNA-editing model by suggesting that UNG functions as a scaffold to recruit repair proteins to the DNA after DNA cleavage caused by an AIDprocessed endonuclease. The role of such an endonuclease is also supported by a further paper from Honjo's group showing that de novo protein synthesis is required for DNA cleavage in CSR.

(Q) References and links

Kirsty Minton

ORIGINAL RESEARCH PAPERS Begum, N. A. et al. Uracil DNA glycosylase activity is dispensable for immunoglobulin class switch. Science 305, 1160-1163 (2004) | Begum, N. A. et al. De novo protein synthesis is required for activation-induced cytidine deaminase-dependent DNA cleavage in immunoglobulin class-switch recombination. Proc. Natl Acad. Sci. USA 101, 13003-13007 (2004)

FURTHER READING Chaudhuri, J. \& Alt, F. W. Class-switch recombination: interplay of transcription, DNA deamination and DNA repair. Nature Rev. Immunol. 4, 541-552 (2004).

\section{IN THE NEWS}

Cannabis can ease MS Cannabis-based treatments could be more effective at treating multiple sclerosis (MS) than originally thought. Patients who participated in a 15-week study - published last year in The Lancet reported that cannabinoid compounds provided relief from some of the painful symptoms of MS, but

independent physiotherapists failed to find hard evidence of reduced muscle stiffness and disability. However, new data presented at the annual meeting of the British Association for the

Advancement of Science in Exeter, UK, indicates that this assessment might have been premature. Of the patients in the initial trial, $80 \%$ opted to continue cannabinoid treatment for up to 1 year, and Dr John Zajicek of the Peninsula Medical School in Plymouth, UK, who headed the study, now says that "In the longer term, there is a suggestion of a more useful beneficial effect, which was not clear at the initial stage" (The Guardian). The extended study indicates that patients who took capsules containing tetrahydrocannabinol (THC), the active ingredient in cannabis, showed some improvements in stiffness and mobility compared with those who took a placebo.

Animal studies have

indicated that cannabinoids slow nerve-cell death; when asked whether this might be responsible for beneficial effects of cannabinoids in patients with MS, Zajicek answered, "Our results are certainly consistent with that hypothesis." (BBC News.)

He also hopes that " ...these results will encourage support of further studies of cannabinoids in MS". In agreement, Dr Philip Robson, Director of the Cannabinoid Research Institute, UK, says that "This is very exciting news and certainly emphasizes the need to assess the potential of cannabis-based products in disease modification as well as symptom relief".

Lucy Bird 